\title{
Desarrollo y medio ambiente. \\ El rol de la bioética en un contexto de \\ cambios ambientales globales
}

\section{Development and environment. Bioethics role in a framework of global environmental changes}

\author{
SOFÍA CASTRO SALVADOR*
}

Resumen: El artículo tiene por objetivo mostrar cómo el modelo de desarrollo dominante ha llevado al planeta a límites insostenibles del medio ambiente. El modelo de desarrollo desde la década de los cincuenta ha estado conducido por el crecimiento económico, sin considerar otras dimensiones como las ambientales y/o sociales. Maddison muestra que tanto la producción, como la población han aumentado considerablemente en el último siglo, pero, a la vez, las brechas han aumentado cada vez más en los bloques regionales, persistiendo los niveles de pobreza y malnutrición en algunos países. Sin embargo, los niveles de producción y consumo han aumentado y ocasionado la crisis energética y ambiental. Estos niveles se han vuelto insostenibles y están llevando al mundo a escenarios catastróficos. Es en este contexto donde la bioética aparece para dar algunas orientaciones sobre cómo deben conducirse los individuos con relación a la vida personal, social y a la naturaleza. Pero estos cambios en la conducta deben ocurrir en el plazo más inmediato ya que ello determinará el futuro del planeta y de la humanidad.

Palabras clave: Desarrollo - producción - consumo - medio ambiente bioética

Summary: The article intends to demonstrate how the dominant development model drives the planet to environmental unsustainable limits. Since the 1950s the development model has been led by economic growth without considering other dimensions as social or environmental. Maddison (2005) explains both production and population have been significantly increased in the last century; however, at the same time regional blocs' gaps have augmented, persisting poverty levels and malnutrition in some countries.

However, production and consumption levels have risen triggering an energetic and environmental crisis. These levels have become unsustainable and are driven the world to catastrophic scenarios. In this context, bioethics rises to give people's guidance concerning personal, social and nature behavior. Anyway, behavior changes should happen in the shortest term since this will shape the future of the planet and humanity.

Keywords: Development - production - consumption - environment bioethics

* Economista, magíster en Desarrollo Ambiental. Profesora del departamento de Economía de la Pontificia Universidad Católica del Perú. Coordinadora de investigación y proyectos del Instituto de Ciencias de la Naturaleza, Territorio y Energías Renovables, INTE-PUCP. 
CONTENIDO: I. EL MODELO DE DESARROLLO IMPERANTE: EL DESARROLLO ECONÓMICO.- II. CRISIS DEL MODELO DE PRODUCCIÓN Y CONSUMO Y SU EFECTO EN EL MEDIO AMBIENTE.- III. LÍMITES DE LA INTERVENCIÓN HUMANA EN LA NATURALEZA.- IV. EL ROL DE LA BIOÉTICA EN LA RELACIÓN DEL HOMBRE CON LA NATURALEZA Y LA CULTURA. UNA NUEVA ÉTICA DEL CONSUMO.- V. REFLEXIONES FINALES.

\section{EL MODELO DE DESARROLLO IMPERANTE: EL DESARROLLO ECONÓMICO}

El término desarrollo refiere a un concepto bastante antiguo y heredero de la noción occidental de progreso. Es en los años cincuenta cuando el término aparece con más regularidad, cuando los organismos internacionales empiezan a utilizarlo para explicar o justificar las brechas existentes entre los países del norte y los países del sur ${ }^{1}$.

El desarrollo, desde entonces, está asociado principalmente con lo económico y en particular con el aumento de la productividad. Los economistas señalan que este último mide la cantidad de producción que una persona puede realizar en una determinada unidad de tiempo, es decir, la productividad — tal como señalan Iguíniz, Ansión y otros«se logra con los medios de trabajo utilizados y con las energías que se obtienen o que se desencadenan en la naturaleza» ${ }^{2}$. El fin último del desarrollo para este modelo es, por lo tanto, aumentar la productividad y esto se logra principalmente a partir de la especialización y de la división del trabajo en el mercado. Lo que importa es que las personas concentren sus esfuerzos en un determinado conjunto de tareas, lo que maximiza a su vez las ganancias y reduce los costos relativos. Samuelson y Nordhaus señalan que «la especialización aumenta la productividad y los niveles de vida» ${ }^{3}$.

A nivel mundial efectivamente se ha producido un crecimiento económico sostenido. El economista norteamericano Angus Maddison realiza un estimado de datos de PIB y población desde inicios del siglo para mostrar que desde 1820 la economía ha crecido históricamente rápido ${ }^{4}$. En la tabla 1 se puede observar que entre 1913 y 2001 la producción en el mundo ha aumentado 13,6 veces, 3,4 veces el tamaño de la población y 4 veces el PIB per cápita. Esto trae abajo la teoría

1 VALCÁRCEL, Marcel. Desarrollo y desarrollo rural: enfoques y reflexiones. Lima: Departamento de Ciencias Sociales-PUCP, 2007, p. 11.

2 IGUíñIZ, Javier \& otros. Desarrollo humano entre el mundo rural y urbano. Lima: Fondo Editorial de la Pontificia Universidad Católica del Perú, 2004, p. 22.

3 SAmuelson, Paul \& William NordHaus. Economía. Decimocuarta edición. Madrid: McGraw Hill, 1993, p. 35.

4 MADDISON, Angus. Growth and Interaction in the World Economy. The Roots of Modernity. Washington: American Enterprise Institute Press, 2005. 
malthusiana que señalaba que el crecimiento de la población aumentaba en forma geométrica, mientras que la producción de alimentos lo hacía en forma aritmética.

La tabla también muestra el crecimiento de las brechas entre los distintos bloques regionales. En el año 1000, Occidente (que incluye, Europa Occidental, Estados Unidos, Canadá, Australia, Nueva Zelanda y Japón) tenía casi el mismo nivel de PIB per cápita que América Latina, a inicios del siglo pasado la relación era de 2,5 a 1 y al finalizar, 3,9 a 1. El crecimiento de este indicador nos muestra, por un lado, la hegemonía económica de los países desarrollados o del norte y, por otro lado, que el aumento de producción en el mundo se puede deber a la necesidad de cubrir las necesidades de una población también en crecimiento.

Tabla 1. Niveles de PIB per cápita, población y PIB mundial y principales regiones, 1000-2001

\begin{tabular}{|c|c|c|c|c|c|c|c|c|}
\hline & 1000 & 1500 & 1820 & 1870 & 1913 & 1950 & 1973 & 2001 \\
\hline \multicolumn{9}{|c|}{ Levels of per-capita GDP (1990 International dollars) } \\
\hline Western Europe & 400 & 771 & 1204 & 1960 & 3458 & 4579 & 11416 & 19256 \\
\hline Western Offshoots & 400 & 400 & 1202 & 2419 & 5233 & 9268 & 16179 & 26943 \\
\hline Japan & 425 & 500 & 669 & 737 & 1387 & 1921 & 11434 & 20683 \\
\hline West & 405 & 702 & 1109 & 1882 & 3672 & 5649 & 13082 & 22509 \\
\hline Asia (excluding Japan) & 450 & 572 & 577 & 550 & 658 & 634 & 1226 & 3256 \\
\hline Latin America & 400 & 416 & 692 & 681 & 1481 & 2506 & 4504 & 5811 \\
\hline E. Europe \& USSR & 400 & 498 & 686 & 941 & 1558 & 2602 & 5731 & 5038 \\
\hline Africa & 425 & 414 & 420 & 500 & 637 & 894 & 1410 & 1489 \\
\hline Rest & 441 & 538 & 578 & 606 & 860 & 1091 & 2072 & 3372 \\
\hline World & 436 & 566 & 667 & 875 & 1525 & 2111 & 4091 & 6049 \\
\hline Interregional spread & $1.1: 1$ & $1.9: 1$ & 2.9:1 & $4.8: 1$ & $8.2: 1$ & $14.6: 1$ & $13.2: 1$ & 18.1:1 \\
\hline West/Rest spread & $0.9: 1$ & $1.3: 1$ & 1.9:1 & $3.1: 1$ & $4.3: 1$ & $5.2: 1$ & $6.3: 1$ & $6.7: 1$ \\
\hline
\end{tabular}

DESARROLLO Y MEDIO AMBIENTE.

EL ROL DE LA BIOÉTICA EN UN CONTEXTO DE CAMBIOS AMBIENTALES GLOBALES

DEVELOPMENT AND

ENVIRONMENT.

BIOETHICS ROLE IN A FRAMEWORK OF GLOBAL

ENVIRONMENTAL CHANGES 


\begin{tabular}{|c|c|c|c|c|c|c|c|c|}
\hline \multicolumn{9}{|c|}{ Population (millons) } \\
\hline Western Europe & 25 & 57 & 133 & 188 & 261 & 305 & 358 & 392 \\
\hline Western Offshoots & 2 & 3 & 11 & 46 & 111 & 176 & 251 & 340 \\
\hline Japan & 8 & 15 & 31 & 34 & 52 & 84 & 109 & 127 \\
\hline West & 35 & 75 & 175 & 268 & 424 & 565 & 718 & 859 \\
\hline Asia (excluding Japan) & 175 & 268 & 679 & 731 & 926 & 1299 & 2140 & 3527 \\
\hline Latin America & 11 & 18 & 22 & 40 & 81 & 166 & 308 & 531 \\
\hline E. Europe \& USSR & 14 & 30 & 91 & 142 & 236 & 267 & 360 & 411 \\
\hline Africa & 32 & 47 & 74 & 90 & 125 & 227 & 390 & 821 \\
\hline Rest & 233 & 363 & 867 & 1004 & 1367 & 1959 & 3198 & 5290 \\
\hline World & 268 & 438 & 1042 & 1272 & 1791 & 2524 & 3916 & 6149 \\
\hline West/World (\%) & 13,0 & 17,2 & 16,8 & 21,1 & 23,7 & 22,4 & 18,3 & 14,0 \\
\hline
\end{tabular}

\section{Levels of GDP (millons of 1990 international dollars)}

\begin{tabular}{|l|r|r|r|r|r|r|r|r|}
\hline Western Europe & 10,2 & 44,2 & 160,1 & 367,6 & 902,3 & 1396 & 4096 & 7550 \\
\hline Western Offshoots & 0,8 & 1,1 & 13,5 & 111,5 & 582,9 & 1635 & 4058 & 9156 \\
\hline Japan & 3,2 & 7,7 & 20,7 & 25,4 & 71,7 & 161 & 1243 & 2625 \\
\hline West & 14,1 & 53,0 & 194,4 & 504,5 & 1556,9 & 3193 & 9398 & 19331 \\
\hline Asia (excluding Japan) & 78,9 & 153,6 & 392,2 & 401,6 & 608,7 & 823 & 2623 & 11481 \\
\hline Latin America & 4,6 & 7,3 & 15 & 27,5 & 119,9 & 416 & 1389 & 3087 \\
\hline E. Europe \& USSR & 5,4 & 15,2 & 62,6 & 133,8 & 367,1 & 695 & 2064 & 2072 \\
\hline Africa & 13,7 & 19,3 & 31,2 & 45,2 & 79,5 & 203 & 550 & 1222 \\
\hline Rest & 102,7 & 195,3 & 501 & 608,2 & 1175,2 & 2137 & 6626 & 17862 \\
\hline World & 116,8 & 248,3 & 695,3 & 1112,7 & 2732,1 & 5330 & 16024 & 37194 \\
\hline West/World (\%) & 12,1 & 21,3 & 28 & 45,3 & 57 & 59,9 & 58,6 & 52 \\
\hline
\end{tabular}

Source: Maddison (2003a, pp. 256-262)

Fuentes: Banco Mundial, 2008c; WRI, 2008 complementado con los datos sobre emisiones provenientes de los cambios en el uso de la tierra de Houghton, 2009.

Nota. Las emisiones de gases de efecto invernadero corresponde a dióxido de carbono $\left(\mathrm{CO}_{2}\right)$, Metano $\left(\mathrm{CH}_{4}\right)$, óxido nitroso $\left(\mathrm{N}_{2} \mathrm{O}\right)$ y gases con alto potencial de contribuir al calentamiento mundial (gases fluorados). Todas ellas se expresan en unidades de dióxido de carbono equivalente $\left(\mathrm{CO}_{2 \mathrm{e}}\right)$, volumen de $\mathrm{CO}_{2}$, que producirá el mismo calentamiento. En 2005, las emisiones provenientes de los cambios en el uso de la tierra en los países de ingreso alto fueron insignificantes. 
La economía y su teoría de la especialización señalaban que la especialización aumenta la productividad y, por lo tanto, conduce a mejores niveles de vida de las personas, sin embargo, la evidencia empírica demuestra que el crecimiento económico no necesariamente conduce a un aumento en la calidad de vida de las personas. Amartya Sen, en su libro Development as Freedom, señala que vivimos en un mundo de contradicciones, por un lado existe opulencia, pero también vivimos en un mundo con grandes privaciones, destitución y opresión. Y además existen aún muchos problemas antiguos, como la persistencia de los niveles de pobreza, insatisfacción de necesidades básicas, hambruna, violación a las libertades más básicas y amenazas crecientes al medio ambiente y a la sostenibilidad de la economía y sociedad. Esto sucede en países ricos como en países pobres 5 .

El crecimiento económico y la agencia de las personas han ayudado a que las situaciones de pobreza en el mundo se reduzcan, aunque aún en algunos lugares estos niveles no se han reducido tanto y persistan en el tiempo a niveles inaceptables. El Banco Mundial señala que en los últimos treinta años, «la proporción de la población en situación de pobreza extrema ha bajado de la mitad a una cuarta parte. Ahora, es mucho menor la proporción de niños malnutridos y con riesgo de muerte prematura. El acceso a la infraestructura moderna está mucho más generalizado» ${ }^{6}$.

El factor decisivo para este cambio positivo según el Banco Mundial es el «rápido crecimiento económico impulsado por la innovación tecnológica y la reforma institucional, en particular en los países de ingreso mediano de nuestros días, donde los ingresos per cápita se han duplicado»?

En los últimos cuatro años, la economía mundial ha sufrido un retroceso debido a la crisis financiera mundial, los países más afectados sorprendentemente son los países desarrollados y algunos países llamados «en desarrollo», a pesar del contexto adverso, han experimentado tasas de crecimiento positivas. De acuerdo a datos estadísticos del Banco Mundial, la tasa de crecimiento económico del mundo para el año 2009 fue de $-0,7 \%$, las economías desarrolladas y la Unión Europea tuvieron un crecimiento negativo de 3,9\% y América Latina y el Caribe, $-1,7 \%$, mientras que el este asiático tuvo $7,1 \%$ de crecimiento y el sur asiático, $6,2 \%{ }^{8}$.

5 Sen, Amartya. Development as Freedom. Nueva York: First Anchor Books, 1999, p. xi.

6 BANCO MUNDIAL. Informe sobre desarrollo mundial 2010. Desarrollo y cambio climático. Washington, 2010, p. 1.

7 L. cit.

8 Ibídem, p. 2. En el 2010, el Banco Mundial muestra que el mundo y todos los bloques obtuvieron tasas de crecimiento positiva.

DESARROLLO Y

MEDIO AMBIENTE.

EL ROL DE LA

BIOÉTICA EN

UN CONTEXTO

DE CAMBIOS

AMBIENTALES

GLOBALES

DEVELOPMENT

AND

ENVIRONMENT.

BIOETHICS ROLE

IN A FRAMEWORK

OF GLOBAL

ENVIRONMENTAL

CHANGES 
Los países que no han sufrido los impactos de esta crisis económica son principalmente países emergentes como India y la China, o como Perú de este lado de la región. Las economías emergentes o las BRIChS (Brasil, Rusia, India, China y Sudáfrica), como se les conoce, han reconfigurado un nuevo orden económico mundial. La mayor producción del mundo proviene de estos países, pero también son países que tienen al interior altos niveles de pobreza y desigualdad, así como en otras regiones del mundo. Todavía existen poblaciones con necesidades básicas insatisfechas y «continúan siendo enormes, y, por primera vez en la historia, este año se ha superado el umbral de los 1.000 millones de personas hambrientas». Estas privaciones obligan a los estados a seguir pensando en la lucha contra estas condiciones adversas, por lo que «el crecimiento y la mitigación de la pobreza continúan siendo la prioridad dominante para los países en desarrollo»?

Previo al período de la crisis financiera del 2008, el mundo experimentó niveles de crecimiento económico global, los cuales vinieron acompañados de altos niveles de consumo. Según el informe sobre el estado del mundo en 2004 publicado por el Instituto Worldwatch, desde la década de 1960 hasta ahora, el consumo se ha cuadriplicado, sin embargo, las personas no son felices, la calidad de vida de la población ha disminuido, sufren más enfermedades, son más vulnerables a riesgos naturales y están expuesto a un deterioro ambiental evidente. «Más de 1700 millones de personas en todo el mundo ingresaron durante gran parte del siglo pasado a la "clase consumista" y adoptaron dietas, sistemas de transporte y estilos de vida hasta ahora limitados a Europa, América del Norte y Japón [...]. El apetito consumidor sin precedentes destruye los sistemas naturales de los que todos dependemos y hace aun más difícil que los pobres satisfagan sus necesidades básicas» ${ }^{10}$.

Cabe señalar que la crisis económica no es la única que experimenta el mundo, a esta hay que agregarle la crisis energética y ambiental y esto afecta especialmente a los países en desarrollo. La población del mundo está consumiendo bienes y servicios a un ritmo insostenible ${ }^{11} \mathrm{y}$ con graves consecuencias para el bienestar de las personas y del planeta. Este comportamiento está provocando cambios ambientales globales, uno de ellos es el cambio climático, que se está presentando como una amenaza sobre todo para los países en desarrollo.

\footnotetext{
9 Ibídem, p. 1.

10 «Crece el consumo en el mundo: más ricos, más gordos, pero no más felices». Clarín (http://edant. clarin.com/diario/2004/01/09/i-02201.htm), 09/01/2004.

11 Actualmente el mundo utiliza el equivalente de un planeta y medio para proveer los recursos que necesitamos y absorber nuestros desechos. El problema es que solo tenemos un planeta. Las proyecciones señalan que, en un escenario moderado, en el 2050 necesitaremos tres planetas (Global Footprint Network. «World Footprint. Do We Fit on the Planet?» (http://www.footprintnetwork. org/en/index.php/gfn/page/world_footprint/), 2008)..
} 


\section{CRISIS DEL MODELO DE PRODUCCIÓN Y CONSUMO Y SU EFECTO EN EL MEDIO AMBIENTE}

En el mundo - $-\mathrm{y}$ en nuestro país en los dos últimos años a propósito de las elecciones presidenciales - se ha discutido mucho sobre el modelo de desarrollo que queremos. Sobre este asunto hay muchos debates académicos y políticos y también muchos deseos sobre el tipo de modelo que debemos tener, un modelo de desarrollo con inclusión social, desarrollo sostenible, un desarrollo humano, etcétera. Lo cierto y lo concreto es que seguimos dominados por el modelo de desarrollo económico que surgió en los años cincuenta como respuesta a la crisis económica de los países después de la segunda guerra mundial ${ }^{12}$. El fin de este modelo, como se dijo en líneas anteriores, es tener altos niveles de productividad, es decir, mientras más se produce, más desarrollado se encuentra un país, sin embargo, esta premisa ha llevado a un agotamiento de los recursos naturales. Por ejemplo, si nuestro país basa su economía en la producción de muebles de madera, el insumo principal es la madera y esto se obtiene talando árboles y quizás sin ningún tipo de manejo racional y sostenible del bosque, lo que puede llevar a la desaparición del mismo.

Una de las críticas al modelo de desarrollo económico es que al aumentar la producción, esta no es distribuida de manera equitativa, es decir, la torta se amplía, pero no se reparte en partes iguales para todos, pero aumentar la producción significa también ejercer una presión sobre los recursos naturales. A partir de la década de los años cincuenta se discute sobre nuevos enfoques de desarrollo que surgen como crítica al modelo anterior. Por ejemplo, el modelo de desarrollo con igualdad surge por la desigual distribución del modelo de desarrollo económico, y el modelo de desarrollo como necesidades básicas surge porque el modelo de desarrollo con igualdad no garantiza que las personas mejoren su calidad de vida. En este contexto es cuando aparecen las preocupaciones por el deterioro del medio ambiente en el mundo. En 1972 se reunieron por primera vez, en Estocolmo, 113 países para discutir sobre cuestiones ambientales internacionales y la situación del medio ambiente, uno de los principales acuerdos fue que estas cumbres se debían realizar cada diez años para hacer una evaluación de la condición del medio ambiente. Esta cumbre concluyó con una Declaración de la Conferencia de las Naciones Unidas sobre el Medio Ambiente Humano que contiene 24 principios sobre el medio ambiente y el desarrollo, así como un plan de acción con 109 recomendaciones.

En el año 1982 la cumbre mundial se realizó en la ciudad de Nairobi. La idea era que esta fuera la Cumbre Oficial de la Tierra, sin embargo, las circunstancias bélicas derivadas de la guerra fría, hicieron fracasar

DESARROLLO Y

MEDIO AMBIENTE.

EL ROL DE LA

BIOÉTICA EN

UN CONTEXTO

DE CAMBIOS

AMBIENTALES

GLOBALES

DEVELOPMENT

AND

ENVIRONMENT.

BIOETHICS ROLE

IN A FRAMEWORK

OF GLOBAL

ENVIRONMENTAL

CHANGES 
cualquier acuerdo. En el año 1987, la Comisión Mundial sobre Ambiente y Desarrollo, liderada por la primera ministra noruega Gro Harlem Bruntland, elaboró el informe «Nuestro futuro común», donde se acuña por primera vez el término desarrollo sostenible y lo define como: «aquel que garantiza las necesidades del presente sin comprometer las posibilidades de las generaciones futuras para satisfacer sus propias necesidades». Años después, la cumbre en Río de Janeiro en 1992 marca un hito importante al establecer la agenda 21 y plantear temas claves como el cambio climático, la biodiversidad y la eliminación de sustancias toxicas.

Una de las cuestiones importantes en la cumbre de Río 92 fue el acuerdo unánime que el modelo de producción y consumo predominante es el que ha llevado al deterioro del medio ambiente:

[...] las principales causas de que continúe deteriorándose el medio ambiente mundial son las modalidades insostenibles de consumo y producción, particularmente en los países industrializados [...]. Aun los niveles actuales de consumo y producción, basados en la superficie productiva media ecológica mundial, superan en un $25 \%$ la capacidad ecológica de la Tierra, lo que significa que incluso a los niveles actuales, la humanidad está gastando el capital natural del planeta a un ritmo considerable ${ }^{13}$.

Esta cumbre se comprometió y estableció convenios en temas como el cambio climático, la diversidad biológica y la lucha contra la desertificación, que luego se ratificaron en protocolos. Protocolos como el de cambio climático en Kyoto, donde los países industrializados se comprometieron a reducir sus niveles de emisión de gases de efecto invernadero (GEI) y se crearon mecanismos de mercado de carbono para compensar estas emisiones a través de los servicios ecosistémicos que los bosques prestan como sumideros de carbono.

En la cumbre mundial de Johannesburgo, en el año 2002, el resultado arrojó que los países no cumplieron con los compromisos acordados en Río 92, con el consecuente aumento del deterioro de los ecosistemas en general. El hombre se convirtió en el principal destructor de su bienestar y de su entorno debido a la gran presión que ejerce sobre los recursos naturales y al parecer le es indiferente la sostenibilidad de los mismos. Es un ser más egoísta, solo piensa en el hoy y no piensa en las necesidades y la satisfacción de las generaciones futuras. 
El Banco Mundial señala que:

[...] ha habido una fuerte relación entre mayores niveles de riqueza y prosperidad y mayor producción de gases de efecto invernadero, pero esta relación no es inevitable. No puede decirse lo mismo de algunos modelos de consumo y producción. Aun cuando se excluyan los países productores de petróleo, las emisiones per cápita en algunos países de ingreso alto son cuatro veces mayores que en otros ${ }^{14}$.

El gráfico siguiente muestra claramente el nivel de emisiones según los niveles de ingreso, a pesar de que los países de ingreso bajo aportan menos GEI, su aporte es importante y sobre todo porque se originan en los cambios en el uso del suelo.

\section{Gráfico 1. Huellas de carbono desiguales: emisiones per cápita en países de ingreso bajo, mediano y alto (2005)}

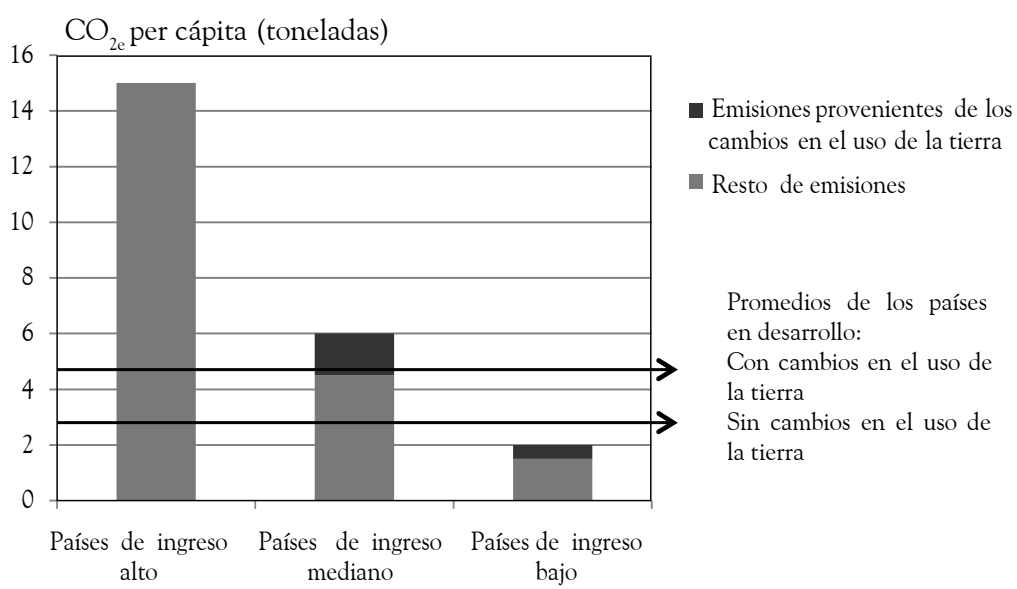

El crecimiento económico de los países, o, como ya se mencionó, los modelos de producción y consumo actuales, están generando más cantidad de $\mathrm{CO}_{2}$, lo que contribuye a un aceleramiento del cambio climático. Al mismo tiempo, la sociedad del conocimiento se expande a límites incalculables, el hombre experimenta sobre otros individuos, sobre animales, sobre las especies vegetales, etcétera, destruye y «crea». Un ejemplo es el de los organismos genéticamente modificados, que se justifican por la escasez de alimentos en el mundo, pero muchos países que generan excedentes de cereales los arrojan al mar, el problema entonces no es de escasez de alimento, sino de distribución de los mismos ${ }^{15}$. 
Sin embargo, ni el crecimiento económico ni el avance científico están mejorando la calidad de vida de las personas, a pesar de la reducción de los niveles de pobreza, estos persisten en muchas regiones. Según el Banco Mundial,

[...] una cuarta parte de la población de los países en desarrollo continúa viviendo con menos de US $\$ 1,25$ al día. Unos 1000 millones de personas carecen de agua potable; 1600 millones, de electricidad, y 3000 millones, de servicios de saneamiento adecuados. La cuarta parte de todos los niños de países en desarrollo están malnutridos ${ }^{16}$.

Además del contexto social de los países en desarrollo aún deprimentes, estos países

soportarán la carga principal de los efectos del cambio climático, al mismo tiempo que se esfuerzan por superar la pobreza y promover el crecimiento económico. Para estos países, el cambio climático representa la amenaza de multiplicar sus vulnerabilidades, erosionar los progresos conseguidos con tanto esfuerzo y perjudicar gravemente las perspectivas de desarrollo ${ }^{17}$.

\section{LÍMITES DE LA INTERVENCIÓN HUMANA EN LA NATURALEZA}

Muchos autores y organismos internacionales, como el Banco Mundial, señalan que, de continuar con el modelo actual de producción de alto consumo de carbono, las emisiones de $\mathrm{CO}_{2}$ se duplicarían a mediados del siglo, lo que llevaría a escenarios catastróficos, debido al aumento de temperatura por encima de los $5^{\circ} \mathrm{C}$. Sin embargo, aún es posible evitar dicho escenario, pero solo si se logra un acuerdo y cambio a través de «una acción mundial concertada para adoptar las políticas adecuadas y tecnologías de bajo consumo de carbono». Con este cambio se podrá lograr tener una trayectoria más sostenible que limite el calentamiento a cerca de $2{ }^{\circ} \mathrm{C}^{18}$.

Los países continúan con el objetivo del desarrollo económico, los compromisos adoptados en Rio 92 no se han cumplido, los niveles de consumo son cada vez mayores, no solo en los países desarrollados, sino también en los desarrollados. Pero el crecimiento económico tiene límites y el umbral lo establece la capacidad de soporte del medio ambiente. Los niveles actuales de producción y consumo son insostenibles, se necesita más de un planeta para cubrir estos niveles, por ello se requiere un cambio y drástico, especialmente en 
[...] la mezcla de energía, de combustibles fósiles a energía renovable y posiblemente energía nuclear, con el uso generalizado de captura y almacenamiento de carbono (CAC). Esto, a su vez, requiere grandes reducciones de costos y la difusión de tecnologías de energía renovable, salvaguardias para contener los residuos nucleares y la proliferación de armas, y adelantos notables en las tecnologías, desde las baterías hasta la captura y almacenamiento de carbono ${ }^{19}$.

Este cambio supone cambios fundamentales en el desarrollo económico, en los estilos de vida, patrones de consumo e imposición de «necesidades». El Banco Mundial señala que la recesión económica puede ser vista como una oportunidad pues puede hacer que «los gobiernos canalicen la inversión y estimulen la energía limpia y eficiente para cumplir los objetivos gemelos de revitalizar el crecimiento económico y mitigar el cambio climático» ${ }^{20}$.

En la última cumbre mundial realizada en Río de Janeiro, denominada Rio + 20, los Estados firmaron el documento «El futuro que queremos», donde se reconoce nuevamente la existencia de modelos insostenibles de producción y consumo, por lo que los países solicitan que «se adopten enfoques globales e integrados del desarrollo sostenible que lleven a la humanidad a vivir en armonía con la naturaleza y conduzcan a la adopción de medidas para restablecer el estado y la integridad del ecosistema de la Tierra» ${ }^{21}$.

La preocupación actual es que uno de los cambios ambientales más importantes, como es el calentamiento global, afecta sobre todo a los países en desarrollo. Por ello la necesidad de adoptar medidas de adaptación al cambio climático en el más inmediato plazo para no empeorar las condiciones de vida de las poblaciones más vulnerables.

\section{EL ROL DE LA BIOÉTICA EN LA RELACIÓN DEL HOMBRE CON LA NATURALEZA Y LA CULTURA. UNA NUEVA ÉTICA DEL CONSUMO}

Como se señaló líneas arriba, los países han estado concentrados en el crecimiento económico, los individuos han producido y consumido sin conciencia de las restricciones del planeta, los ecosistemas se destruyen cada vez más y en general nuestra actitud frente al medio ambiente ha sido de desidia y de indiferencia.

La ciencia y la tecnología se han desarrollado rápidamente y se posicionan en el imaginario colectivo como la única manera para

19 L. cit.

20 Ibídem, p. 190.

21 Organización DE LAS NACIONES UNIDAS. "El futuro que queremos. Documento final de la Conferencia». Rio+20 Conferencia de las Naciones Unidas sobre el Desarrollo Sostenible (https://rio20.un.org/sites/rio20.un.org/files/a-conf.216-l-1_spanish.pdf.pdf) 2012, p. 8.

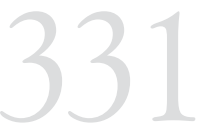

DESARROLLO Y

MEDIO AMBIENTE.

EL ROL DE LA

BIOÉTICA EN

UN CONTEXTO

DE CAMBIOS

AMBIENTALES

GLOBALES

DEVELOPMENT

AND

ENVIRONMENT.

BIOETHICS ROLE

IN A FRAMEWORK

OF GLOBAL

ENVIRONMENTAL

CHANGES 
otorgar bienestar a las poblaciones. El sentido de la sociedad dependerá de la manera ética como se construya el conocimiento y en segundo lugar de sus aplicaciones prácticas. Por ello es importante saber cuáles son los límites del hombre para intervenir en la naturaleza sin dañarla ni interferir en sus interrelaciones. Este avance tecnológico le da al hombre poder económico y político. Nunca como ahora, una simple criatura, como el hombre, ha podido acceder a tanto poder destructor, gracias al conocimiento convertido en demencia ${ }^{22}$.

En un mundo donde la población cada vez crece más, la sociedad se ha vuelto una sociedad consumista de bienes inútiles en un «planeta que no tiene cómo crecer por que su existencia es limitada y finita» ${ }^{23}$. Estos problemas necesitan de respuestas éticas, requieren de un cambio en la visión y el sentido del mundo, en la conducta del individuo con relación a la vida personal, social y a la naturaleza y la producción de bienes y consumo ${ }^{24}$. La bioética en este sentido, aparece para dar directrices, pistas a los individuos sobre cómo conducirse de manera individual y en colectivo frente al medio ambiente. El origen de la bioética, en los años cincuenta, está relacionado con la medicina ${ }^{25}$ y es recién en la década de los setenta, cuando en América Latina se empieza a discutir en el contexto de una moral reconciliadora del hombre con la naturaleza y su cultura.

Para Cely la bioética es «el puente para el diálogo entre todas las ciencias, es el lenguaje conciliador de intereses, que lleva a consensos comprometidos con el cuidado que los individuos hagamos de nosotros y de nuestra casa, para bien de la actual y futuras generaciones» ${ }^{26}$.

La bioética nos dice que debemos actuar ahora pensando en nuestras necesidades, en nuestro entorno, pero también pensando en el futuro, «porque lo que hagamos hoy determinará el clima de mañana y las opciones que configurarán nuestro futuro» ${ }^{27}$.

La sociedad debe cambiar las preguntas tradicionales de la economía de la producción, como qué y cuánto producir por las preguntas sobre el consumo, iqué se consume? iquién consume? y iquién decide lo que se consume? La bioética nos permite repensar en este aspecto sobre el consumo de necesidades básicas y no de bienes superfluos, en general, nos hace pensar en lo que consumimos para no seguir destruyendo ${ }^{28}$.

22 Cely, Gilberto (ed.). Ob. cit., pp. 19-24.

23 TEALDI, Juan Carlos. Diccionario latinoamericano de bioética. Bogotá: Unesco/Red Latinoamericana y del Caribe de Bioética/Universidad Nacional de Colombia, 2008, p. 465.

24 L. cit.

25 lbídem.

26 Cely, Gilberto (ed.). Ob. cit., p. 24.

27 BANCO MUNDIAL. Ob. cit., p. v.

28 CortinA, Adela. Consumo...luego existo. Intervención transcrita de Adela Cortina en una mesa redonda que sobre el tema del consumo organizó Cristianisme i Justícia en mayo de 2003.. 


\section{V.REFLEXIONES FINALES}

El modelo de producción y consumo ha llevado al planeta a una crisis ambiental global. El modelo de desarrollo actual no es compatible con la protección y conservación de los recursos naturales, al contrario, hay un consumo que sobrepasa los límites del planeta, consumimos como si tuviéramos otros planetas disponibles. Problemas persistentes, como la hambruna y la pobreza, son ahora compartidos por la crisis ambiental. Los países en desarrollo, además de luchar contra la pobreza y desigualdad, también deben hacer frente a los embates del cambio climático.

Las Organización de las Naciones Unidas, en su última cumbre Río +20, alerta de los escenarios catastróficos que nos deparan si es que seguimos en la misma senda de crecimiento. El Banco Mundial, en su informe del año 2010 sobre desarrollo y cambio climático, también hace un llamado a la acción y a una acción concertada, ya que este no es un problema de un solo país o un bloque, es un tema de todos.

El problema del cambio climático no puede resolverse si los países no cooperan a escala mundial para mejorar la eficiencia energética, desarrollar y desplegar tecnologías limpias, y ampliar los sumideros naturales que permitan absorber gases y proteger el medio ambiente. Debemos proteger la vida humana y los recursos ecológicos. Debemos actuar de común acuerdo y de un modo diferenciado y equitativo ${ }^{29}$.

Asimismo, el Banco Mundial señala que el éxito no se debe solo a lo que los Estados implementen, sino principalmente a la conducta individual de los ciudadanos.

Los individuos, en tanto ciudadanos y consumidores, determinarán el futuro del planeta. Si bien un creciente número de personas sabe sobre el cambio climático y cree necesario adoptar medidas, son muy pocos los que lo consideran una prioridad y demasiados los que no actúan cuando la oportunidad se presenta. En consecuencia, el mayor desafío reside en modificar comportamientos e instituciones, en particular en los países de ingreso alto. Es necesario introducir cambios en las políticas públicas (locales, regionales, nacionales e internacionales) para facilitar y hacer más atractiva la acción de empresas y ciudadanos ${ }^{30}$.

DEVELOPMENT

ENVIRONMENT.

BIOETHICS ROLE

IN A FRAMEWORK

OF GLOBAL

ENVIRONMENTAL 